\title{
Small regular graphs of girth 7
}

\author{
M. Abreu *† \\ Dipartimento di Matematica, \\ Informatica ed Economia \\ Università degli Studi della Basilicata \\ I-85100 Potenza, Italy \\ marien.abreu@unibas.it \\ Departament de Matemt́ica Aplicada III \\ Universitat Politècnica de Catalunya \\ E-08034 Barcelona, Spain. \\ m. camino. balbuena@upc.edu

$$
\text { D. Labbate }{ }^{* \dagger}
$$ \\ Dipartimento di Matematica, \\ Informatica ed Economia \\ Università degli Studi della Basilicata \\ I-85100 Potenza, Italy \\ domenico.labbate@unibas.it
}

\author{
G. Araujo-Pardo * \\ Instituto de Matemáticas \\ Universidad Nacional Autónoma de México \\ Juriquilla 76230 \\ Querétaro, México \\ garaujo@matem. unam.mx
}

\section{Balbuena $* \S$}

Matemáticas Aplicadas y Sistemas Universidad Autónoma Metropolitana Unidad de Cuajimalpa Col. Santa Fé, Cuijimalpa, 05348, México

\section{J. Salas}

Departament d'Enginyeria, Informàtica i Matemàtiques Universitat Rovira i Virgili 43007 Tarragona, Spain julian.salas@urv.cat

Submitted: Mar 19, 2014; Accepted: Jun 17, 2015; Published: Jul 1, 2015

Mathematics Subject Classifications: 05C35, 51E12

\begin{abstract}
In this paper, we construct new infinite families of regular graphs of girth 7 of smallest order known so far. Our constructions are based on combinatorial and geometric properties of $(q+1,8)$-cages, for $q$ a prime power. We remove vertices from such cages and add matchings among the vertices of minimum degree to achieve regularity in the new graphs. We obtain $(q+1)$-regular graphs of girth 7 and order $2 q^{3}+q^{2}+2 q$ for each even prime power $q \geqslant 4$, and of order $2 q^{3}+2 q^{2}-q+1$ for each odd prime power $q \geqslant 5$.
\end{abstract}

Keywords: Cages, girth, incidence graph

\footnotetext{
${ }^{*}$ Research supported by CONACyT-México under projects 178395, and PAPIIT-México under projects IN101912.

${ }^{\dagger}$ Research supported by the Italian Ministry MIUR and carried out within the activity of INdAM-GNSAGA.

${ }^{\ddagger}$ Research supported by CONACyT-México under projects 166306, and PAPIIT-México under projects IN104915.

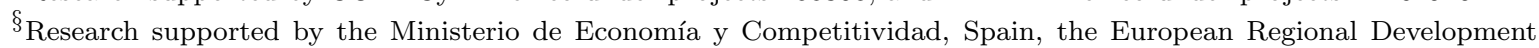
Fund (ERDF) under project MTM2014-60127-P; and under the Catalonian Government project 1298 SGR2009.

『Partial support by the Spanish Government through project ICWT (TIN2012-32757)
} 


\section{Introduction}

Throughout this paper, only undirected simple graphs without loops or multiple edges are considered. For terminology and notation not explicitly defined here, please refer to [14]. Let $G$ be a graph with vertex set $V=V(G)$ and edge set $E=E(G)$. We denote the subgraph of $G$ induced by a subset $U \subset V(G)$ as $G[U]$, and it is the graph with $V(G[U])=U$ and for any $u, v \in V(G[U])$ the edge $u v$ belongs to $E(G[U])$ if and only if $u v \in E(G)$. The girth of a graph $G$ is the number $g=g(G)$ of edges in a smallest cycle. For every $v \in V, N_{G}(v)$ denotes the neighbourhood of $v$, that is, the set of all vertices adjacent to $v$, we may denote it simply by $N(v)$. Similarly, for each positive integer $t$ we denote by $N_{t}(v)$ the neighborhood of $v$ at distance $t$, i.e. the set $N_{t}(v)=\{x \in V(G): d(x, v)=t\}$, and the neighborhood of an edge $u v$ at distance $t$ is the set $N_{t}(u v)=\{x \in V(G): d(x, u)=t$ or $d(x, v)=t\}$.

The degree of a vertex $v \in V$ is the cardinality of $N(v)$. A graph is called regular if all the vertices have the same degree. A $(k, g)$-graph is a $k$-regular graph with girth $g$. Erdös and Sachs [16] proved the existence of $(k, g)$-graphs for all values of $k$ and $g$ provided that $k \geqslant 2$. Thus most work carried out has focused on constructing a smallest one $[1,3,4,5,6,7,8,9,10,11,15,17,19,20,22,23,27,28,31] . \mathrm{A}(k, g)$-cage is a $k$ regular graph with girth $g$ having the smallest possible number of vertices $n(k, g)$. Cages have been studied intensely since they were introduced by Tutte [35] in 1947, and their construction is a very difficult task.

Counting the numbers of vertices in the distance partition with respect to a vertex yields Moore's lower bound $n_{0}(k, g)$ (cf. e.g. [18, Eq. (2)]) with the precise form of the bound depending on whether $g$ is even or odd:

$$
n_{0}(k, g)= \begin{cases}1+k+k(k-1)+\cdots+k(k-1)^{(g-3) / 2} & \text { if } g \text { is odd; } \\ 2\left(1+(k-1)+\cdots+(k-1)^{g / 2-1}\right) & \text { if } g \text { is even. }\end{cases}
$$

Biggs [12] calls the excess of a $(k, g)$-graph $G$ the difference $|V(G)|-n_{0}(k, g)$. The construction of graphs with small excess is also quite challenging. Biggs is the author of a report on distinct methods for constructing cubic cages [13]. More details about constructions of cages can be found in the survey by Wong [39] or in the book by Holton and Sheehan [21] or in the more recent dynamic cage survey by Exoo and Jajcay [18].

A $(k, g)$-cage with $n_{0}(k, g)$ vertices and even girth exists only when $g \in\{4,6,8,12\}$ [19]. If $g=4$ they are the complete bipartite graph $K_{k, k}$, and for $g=6,8,12$ these graphs are the incidence graphs of generalized $g / 2$-gons of order $k-1$. This is the main reason for $(k, g)$-cages with $n_{0}(k, g)$ vertices and even girth $g$ are called generalized polygon graphs [12]. In particular a 3-gon of order $k-1$ is also known as a projective plane of order $k-1$. The 4-gons of order $k-1$ are called generalized quadrangles of order $k-1$, and, the 6-gons of order $k-1$, generalized hexagons of order $k-1$. All these objects are known to exist for all prime power values of $k-1$, and no example is known when $k-1$ is not a prime power (cf. e.g. [30, p.25], [34]).

On the other hand, a general upper bound for $n(k, g)$ has been given by Sauer (cf. 
e.g. [18, Thm. 7], [33]) and it states that for every $k \geqslant 2$ and $g \geqslant 3$

$$
n(k, g) \leqslant \begin{cases}2(k-2)^{(g-2)} & \text { if } g \text { is odd; } \\ 4(k-1)^{(g-3)} & \text { if } g \text { is even. }\end{cases}
$$

In particular, for $g=7$, Sauer's bound has been the best known so far, and it gives that $n(k, 7) \leqslant 2(k-2)^{5}$.

In this paper, we construct new infinite families of small regular graphs of girth 7 . Our constructions are based on combinatorial and geometric properties of $(q+1,8)$-cages, for $q$ a prime power, which are summarized in Section 2. We remove vertices from such cages and add matchings among the vertices of minimum degree to achieve regularity in the new graphs (cf. Definitions 2 and 9). In Section 3 we construct $(q+1,7)$-graphs of order $2 q^{3}+q^{2}+2 q$ for each even prime power $q \geqslant 4$ (cf. Construction 1 and Theorem 7 ). In Section 4 we construct $(q+1,7)$-graphs of order $2 q^{3}+2 q^{2}-q+1$ for each odd prime power $q \geqslant 5$ (cf. Construction 2 and Theorem 15). All these graphs are the smallest $(q+1,7)$-graphs known so far, for each prime power $q>5$.

Indeed, for $q=4$ our graph matches the order of the known $(5,7)$-graphs of order $152[26,32]$. For $q=5$ there are smaller known (6,7)-graphs of order 294 [26, 32] (our $(6,7)$-graph has 296 vertices). However, for prime powers $q>5$, our graphs improve Sauer's upper bound (2), and their order lies within a constant factor of Moore's lower bound which equals $(1+o(1)) q^{3}$. Specifically, we prove that

$$
n(k, g) \leqslant \begin{cases}2(k-1)^{3}+(k-1)^{2}+2(k-1) & \text { for each even prime power } k-1 \geqslant 4 ; \\ 2(k-1)^{3}+2(k-1)^{2}-(k-2) & \text { for each odd prime power } k-1 \geqslant 5 .\end{cases}
$$

\section{Preliminaries}

It is well known $[24,30]$ that $Q(4, q)$ and $W(3, q)$ are the only two classical generalized quadrangles with parameters $s=t=q$.

The generalized quadrangle $W(3, q)$ is the dual generalized of $Q(4, q)$, and they are selfdual for $q$ even.

In 1966 Benson [11] constructed $(q+1,8)$-cages from the generalized quadrangle $Q(4, q)$. He defined the point/line incidence graph $\Gamma_{q}$ of $Q(4, q)$ which is a $(q+1)$ regular graph of girth 8 with $n_{0}(q+1,8)$ vertices, hence $\Gamma_{q}$ is a $(q+1,8)$-cage. Note that, $\Gamma_{q}$ is also the point/line incidence graph of $W(3, q)$.

For any generalized quadrangle $Q$ of order $(s, t)$ and every point $x$ of $Q$, let $x^{\perp}$ denote the set of all points collinear with $x$. For a nonempty set $X$ of vertices of $Q$, we define $X^{\perp}:=\bigcap_{x \in X} x^{\perp}$. Note that $N_{2}(x)$ in the incidence graph $\Gamma_{q}$, corresponds in the geometry to $x^{\perp}$ for a point $x \in Q$.

The span of the pair $(x, y)$ is $s p(x, y)=\{x, y\}^{\perp \perp}=\left\{u \in P: u \in z^{\perp} \forall z \in x^{\perp} \cap y^{\perp}\right\}$, where $P$ denotes the set of points in $Q$. If $x$ and $y$ are not collinear, then $\{x, y\}^{\perp \perp}$ is also called the hyperbolic line through $x$ and $y$. If the hyperbolic line through two noncollinear points $x$ and $y$ contains precisely $t+1$ points, then the pair $(x, y)$ is called regular. A 
point $x$ is called regular if the pair $(x, y)$ is regular for every point $y$ not collinear with $x$. It is important to recall that the concept of being regular also exists for a graph. Hence, we will emphasize when the word "regular" refers to a point of a geometry or to a graph.

Remark 1. [30, p.33, dual of 3.3.1(i)] Every point in $W(q)$ is regular (i.e. $|s p(x, y)|=q+1$ for all non-collinear $x, y)$.

There are several equivalent coordinatizations of these generalized quadrangles (cf. [29], [36], [37], see also [24]) each giving a labeling for the graph $\Gamma_{q}$. In Section 4 we present a further labeling of $\Gamma_{q}$, equivalent to previous ones (cf. $[1,2]$ ), which will be central for our constructions since it allows us to keep track of the properties (such as regularity and girth) of the small regular graphs of girth 7 obtained from $\Gamma_{q}$.

\section{Construction of small $(q+1,7)$-graphs for even prime powers}

In this section we construct a family of $(q+1,7)$-graphs of order $2 q^{3}+q^{2}+2 q$ obtained from a $(q+1,8)$-cage $\Gamma_{q}$ for each even prime power $q \geqslant 4$. In general terms, we proceed by removing, from a $(q+1,8)$-cage, a subgraph $H$ consisting of a distinguished vertex $x$, its neighbours, and almost all its second neighbours (the neighbourhoods of all but two of the neighbours of $x$ ). The resulting graph is not regular, indeed the neighbours of the subgraph $H$ in the cage, are left with degree $q$. So we add appropriate matchings among such vertices to restore the $(q+1)$-regularity of the graph. The constructed graph has girth at most 7 by Equation (1). The details in this Section are devoted to choosing the matchings in an appropriate way to obtain girth exactly 7 in the graph.

Let $x \in V\left(\Gamma_{q}\right)$ and $N(x)=\left\{x_{0}, \ldots, x_{q}\right\}$. Label $X_{i}=N\left(x_{i}\right)-x=\left\{x_{i 1}, \ldots, x_{i q}\right\}$ for all $i \in\{0, \ldots, q\}$. We denote $X_{i j}=N\left(x_{i j}\right) \backslash\left\{x_{i}\right\}$ for $i \in\{0, \ldots, q\}$ and $j \in\{1, \ldots, q\}$ and observe that the sets $X_{i j}$ have even cardinality. Let $\mathcal{Z}=\left\{X_{0}, X_{1}, X_{i j}: i=2, \ldots, q, j=\right.$ $1, \ldots, q\}$. For each set $Z \in \mathcal{Z}, M_{Z}$ will denote a perfect matching of $Z$. Let

$$
H=N(x) \cup \bigcup_{i=2}^{q} N\left(x_{i}\right) \subset V\left(\Gamma_{q}\right)
$$

To obtain a small regular graph of girth 7 , we consider the graph $\Gamma_{q}-H$ and then add matchings $M_{Z}$ between the remaining neighbors of the vertices in $H$.

Definition 2. Let $\Gamma_{q}$ be a $(q+1,8)$-cage for an even prime power $q \geqslant 4$ and $H$ as in (4). We define $\Gamma_{q}^{1}$ to be the graph with: $V\left(\Gamma_{q}^{1}\right):=V\left(\Gamma_{q}-H\right)$ and $E\left(\Gamma_{q}^{1}\right):=E\left(\Gamma_{q}-H\right) \cup \bigcup_{Z \in \mathcal{Z}} M_{Z}$.

Remark 3. The graph $\Gamma_{q}^{1}$ has order $\left|V\left(\Gamma_{q}\right)\right|-\left(q^{2}+2\right)$ and all its vertices have degree $q+1$. Furthermore, the girth of $\Gamma_{q}^{1}$ is at most 7 by Equation (1).

Remark 4. Let $u$ and $v$ be distinct vertices of a graph $G$ of girth 8 such that there is a $u v$-path $P$ of length $t<8$. Then every $u v$-path $P^{\prime}$ such that $E(P) \cap E\left(P^{\prime}\right)=\emptyset$ has length $\left|E\left(P^{\prime}\right)\right| \geqslant 8-t$. 
Proposition 5. Let $\Gamma_{q}$ be a $(q+1,8)$-cage for an even prime power $q \geqslant 4$ and $\Gamma_{q}^{1}$ as in Definition 2. Then $\Gamma_{q}^{1}$ has girth 7 if the following condition holds:

For each $u v \in M_{X_{i j}}$ and each $X_{k l}$, where $i, k \in\{0, \ldots, q-2\}, j, l \in\{1, \ldots, q\}$

$$
E\left(\Gamma_{q}^{1}\left[N_{2}(u v) \cap X_{k l}\right]\right) \cap M_{X_{k l}}=\emptyset .
$$

Proof. By Remark 3 the graph $\Gamma_{q}^{1}$ has girth at most 7 . From Remark 4, the distances in $\Gamma_{q}-H$ between the elements in the sets $Z \in \mathcal{Z}$ satisfy the following:

(i) If $u, v \in Z$, then $d_{\Gamma_{q}-H}(u, v) \geqslant 6$ because they have a common neighbor $z$ in $\Gamma_{q}$.

(ii) If $u \in X_{0}$ and $v \in X_{1}$, then $d_{\Gamma_{q}-H}(u, v) \geqslant 4$.

(iii) If $u \in X_{i}$ and $v \in X_{k j}$, then $d_{\Gamma_{q}-H}(u, v) \geqslant 3$, for $i \in\{0,1\}, k \in\{2, \ldots, q\}$ and $j \in\{1, \ldots, q\}$.

(iv) If $u \in X_{l j}$ and $v \in X_{l k}$, then $d_{\Gamma_{q}-H}(u, v) \geqslant 4$, for $l \in\{2, \ldots, q\}$ and $j, k \in\{1, \ldots, q\}$.

(v) If $u \in X_{t j}$ and $v \in X_{l k}$, then $d_{\Gamma_{q}-H}(u, v) \geqslant 2$, for $t \neq l, t, l \in\{2, \ldots, q\}$ and $j, k \in\{1, \ldots, q\}$.

Let $C$ be a shortest cycle in $\Gamma_{q}^{1}$. If $E(C) \subset E\left(\Gamma_{q}-H\right)$, then $|C| \geqslant 8$. Suppose $C$ contains edges in $M=\bigcup_{Z \in \mathcal{Z}} M_{Z}$. If $C$ contains exactly one such edge, then by (1), $|C| \geqslant 7$. If $C$ contains exactly two edges $e_{1}, e_{2} \in M$, we have the following cases:

- If both $e_{1}, e_{2}$ lie in the same $M_{Z}$, then by (i), $|C| \geqslant 14>7$.

- If $e_{1} \in M_{X_{0}}$ and $e_{2} \in M_{X_{1}}$, then $|C| \geqslant 10>7$ by (ii).

- If $e_{1} \in M_{X_{i}}, i=0,1$, and $e_{2} \in M_{X_{k j}}$, then $|C| \geqslant 8>7$ by (iii).

- If $e_{1} \in M_{X_{l j}}$ and $e_{2} \in M_{X_{l k}}$, then $|C| \geqslant 10>7$ by (iv).

- If $e_{1} \in M_{X_{t j}}$ and $e_{2} \in M_{X_{l k}}$, then $|C| \geqslant 7$ for $t \neq l$, by condition $(*)$.

If $C$ contains at least three edges of $M$, then $|C| \geqslant 9>7$ since $d_{\Gamma_{q}}(u, v) \geqslant 2$ for all $u, v \in\left\{X_{0}, X_{1}, X_{i j}\right\}$ with $i \in\{2, \ldots, q\}$ and $j \in\{1, \ldots, q\}$ by (i)-(v). From the above and Remark 3 it follows that $\Gamma_{q}^{1}$ has girth 7 .

Lemma 6. There exist $q^{2}-q$ matchings $M_{X_{i j}}$ satisfying condition (*) in Proposition 5. Proof. Let $\Omega_{j}:=\bigcap_{i=2}^{q} N\left(X_{i j}\right)$ for $j=1, \ldots, q$. Let $w_{j 1} \in \Omega_{j}$. As $d_{\Gamma_{q}}\left(x, w_{j 1}\right)=4$ there exist other $q-1$ elements in $\Omega_{j}$ mutually at distance four, since $\Gamma_{q}$ is the incidence graph of a generalized quadrangle $W(q)$. Label $\Omega_{j}=\left\{w_{j 1}, \ldots, w_{j q}\right\}$. 


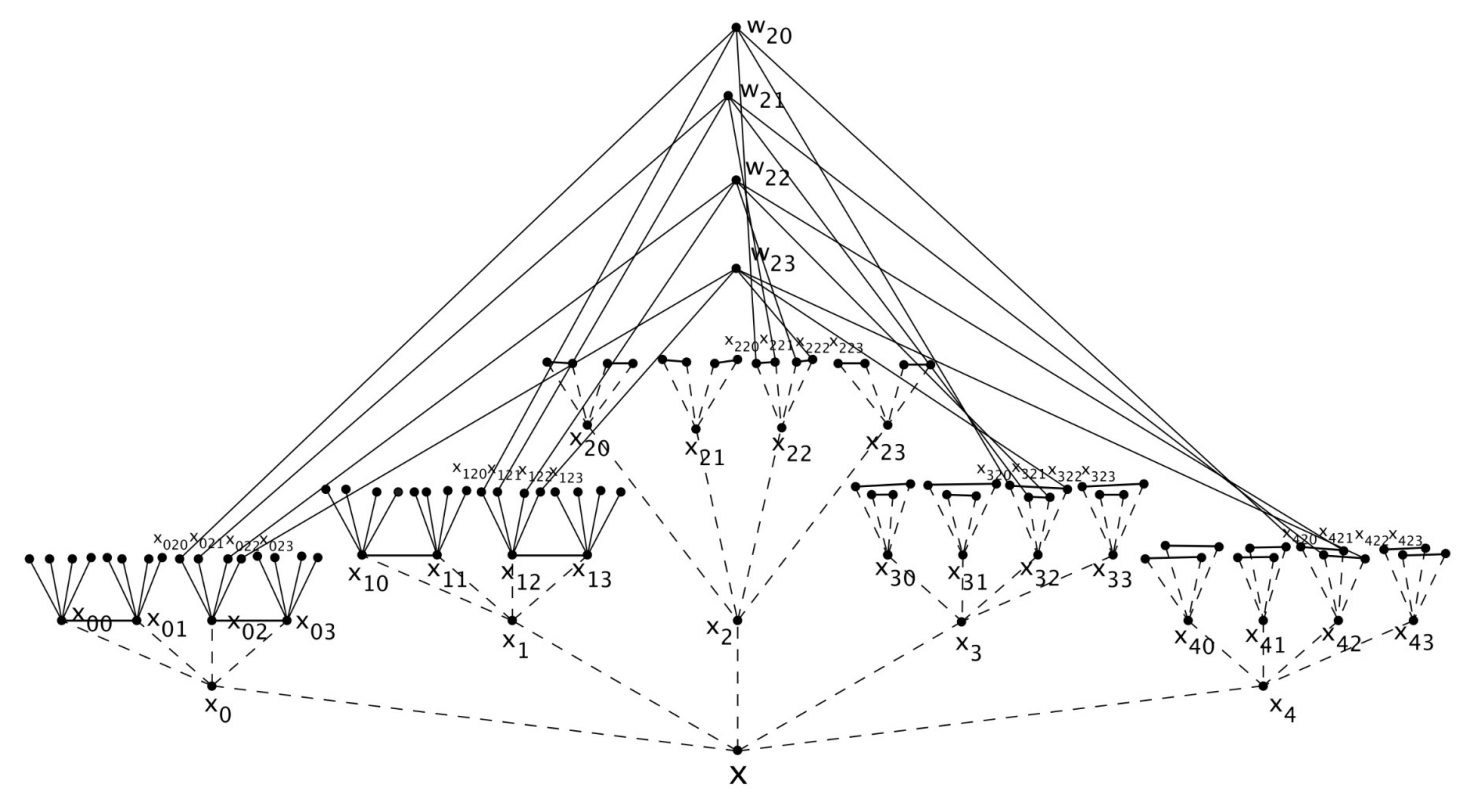

Figure 1: The dashed edges and their lower end-vertices illustrate the subgraph $H$, removed from $\Gamma_{q}$, when $q=4$. Above, find the choice of the matchings that lead to a $(5,7)$-graph of girth exactly 7 and order 152 .

Every vertex $w_{j h}$ is adjacent to exactly one vertex in $X_{i j}$ since the girth of $\Gamma_{q}$ is 8 . Denote $\left\{x_{i j h}\right\}=N\left(X_{i j}\right) \cap N\left(w_{j h}\right)$ for each $i \in\{2, \ldots, q\}$ and $j, h \in\{1, \ldots, q\}$ (see Figure 1). Note that $x_{i j h}$ is well labeled, because if $x_{i j h}$ had two neighbors $w_{j h}, w_{j h^{\prime}} \in \bigcap_{i=2}^{q} N\left(X_{i j}\right)$, then $\Gamma_{q}$ would contain the cycle $x_{i j h} w_{j h^{\prime}} x_{i^{\prime} j h^{\prime}} x_{i^{\prime} j} x_{i^{\prime} j h} w_{j h}$ of length 6 .

Therefore, take the complete graph $K_{q}$ label its vertices as $h \in\{1, \ldots, q\}$. We know that it has a 1 -factorization with $q-1$ factors $F_{1}, \ldots, F_{q-1}$ since $q$ is even (cf. e.g. [38]). For each $i=2, \ldots, q$, let $x_{i j h} x_{i j h^{\prime}} \in M_{X_{i j}}$ if and only if $h h^{\prime} \in F_{i-1}$.

To prove that the matchings $M_{X_{i j}}$ defined in this way fulfill condition (*), suppose that $x_{i j h} x_{i j h^{\prime}} \in M_{X_{i j}}$ and $x_{i^{\prime} j h} x_{i^{\prime} j h^{\prime}} \in M_{X_{i^{\prime} j}}$ for $i^{\prime} \neq i$. Then $F_{i}$ and $F_{i^{\prime}}$ would have the edge $h h^{\prime}$ in common contradicting that they come from a 1-factorization.

To conclude, notice that for $u v \in M_{X_{i j}}$ and $a, b \in X_{k l}$ with $l \neq j$ and possibly $k=i$, the distances $d(u, a)$ and $d(v, b)$ are at least 4 .

Therefore, there exist $q^{2}-q$ matchings $M_{X_{i j}}$ with the desired property.

Construction 1: Let $q \geqslant 4$ be an even prime power. Let $\Gamma_{q}^{1}$ be the $(q+1)$-regular graph of order $2 q^{3}+q^{2}+2 q$ from Definition 2 with $M_{X_{i j}}$ as in the proof of Lemma 6 , for $i \in\{2, \ldots, q\}$ and $j \in\{1, \ldots, q\}$; and with $M_{X_{0}}$ and $M_{X_{1}}$ matchings of $X_{0}$ and $X_{1}$ respectively, chosen arbitrarily. Then, the graph $\Gamma_{q}^{1}$ obtained with such a choice of matchings has girth 7 by Proposition 5 .

As a consequence we have the following theorem. 
Theorem 7. Let $q \geqslant 4$ be an even prime power. Then, there is a $(q+1)$-regular graph of girth 7 and order $2 q^{3}+q^{2}+2 q$.

Figure 1 illustrates this construction for $q=4$. Note that this $(5,7)$-graph has 152 vertices, as the two found in 2001 by McKay and Yang [26, 32].

\section{Constructions of small $(q+1,7)$-graphs for an odd prime power.}

In this section we construct an infinite family of $(q+1,7)$-graphs of order $2 q^{3}+2 q^{2}-q+1$ for odd prime power $q \geqslant 5$. Analogously to Section 3, we will delete a set $H$ of vertices from a $(q+1,8)$-cage $\Gamma_{q}$ and add matchings $M_{Z}$ between the remaining neighbors of $H$ to obtain a small regular graph of girth 7 . In general terms, the subgraph $H$ consists of two distinguished vertices $x$ and $y$ at distance 4 in $\Gamma_{q}$; their neighbours and all but three of the common second neighbours of $x$ and $y$ (see Figure 2). The removal of $H$ from $\Gamma_{q}$ leaves a non regular graph. Thus, we add appropriate matchings among the vertices of lesser degree, and three sporadic 2-paths, to restore the $(q+1)$-regularity of the graph (cf. Definition 9). The constructed graph has girth at most 7 by Equation (1). As in Section 3, the details that follow are devoted to choosing the matchings in an appropriate way to obtain girth exactly 7 in the graph. In particular, some of the matchings can be chosen combinatorially, as for the even case (cf. Lemma 12). However, for the remaining ones, we rely on an algebraic coordinatization of the $(q+1,8)$-cage (cf. Definition 13 and Lemma 14).

Specifically, the set $H$ and matchings $M_{Z}$, are defined as follows.

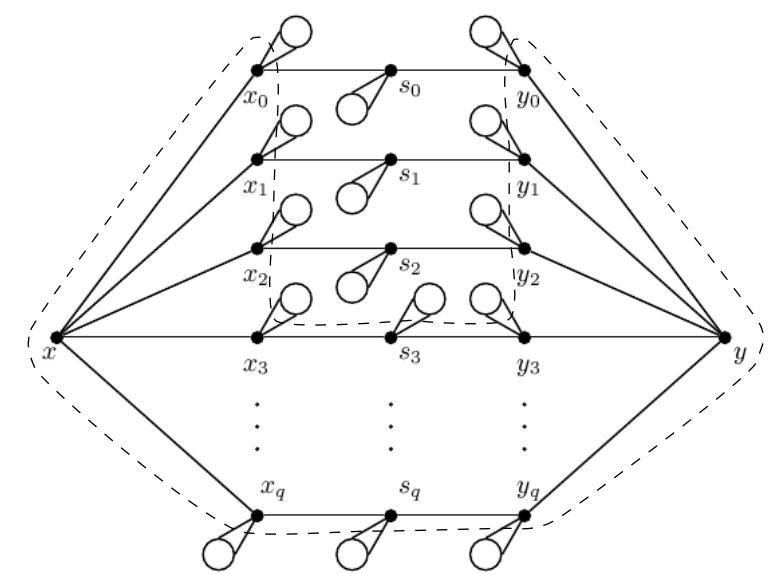

Figure 2: Subgraph of $\Gamma_{q}$ used to define $H$ and $\mathcal{Z}$. The subgraph $H$ is highlighted by the dashed line. 
Definition 8. Let $x, y \in V\left(\Gamma_{q}\right)$ be vertices at distance 4 in $\Gamma_{q}$, and let $x x_{i} s_{i} y_{i} y$ be the internally (vertex) disjoint $x y$-paths for $i=0, \ldots, q$ (which exist since $\Gamma_{q}$ is $(q+1)$ connected, see [25]). We define the following sets (see Figure 2):

$$
\begin{aligned}
& H=\left\{x, y, s_{3}, s_{4}, \ldots, s_{q}\right\} \cup N(x) \cup N(y) \subset V\left(\Gamma_{q}\right) ; \\
& X_{i}=N\left(x_{i}\right) \cap V\left(\Gamma_{q}-H\right), \quad i=0, \ldots, q ; \\
& Y_{i}=N\left(y_{i}\right) \cap V\left(\Gamma_{q}-H\right), \quad i=0, \ldots, q ; \\
& S_{i}=N\left(s_{i}\right) \cap V\left(\Gamma_{q}-H\right), \quad i=3, \ldots, q .
\end{aligned}
$$

Notice that the vertices of $\Gamma_{q}-H$ have degrees $q-1, q$ and $q+1$. The vertices $s_{0}, s_{1}, s_{2}$ have degree $q-1$, those in $X_{i} \cup Y_{i} \cup S_{i}$ have degree $q$ and all the remaining vertices of $\Gamma_{q}-H$ have degree $q+1$. Therefore, in order to obtain a $(q+1)$-regular graph, we need to add edges to $\Gamma_{q}-H$ in such a way that cycles of length smaller than 7 are avoided.

Similarly as before, let $\mathcal{Z}$ be the family of all the sets $X_{i}, Y_{i}, S_{i}$. Note that, all sets in $\mathcal{Z}$ have even cardinality. For each $Z \in \mathcal{Z}, M_{Z}$ will denote a perfect matching of $V(Z)$.

Definition 9. Let $\Gamma_{q}$ be a $(q+1,8)$-cage for odd prime power $q \geqslant 5$.

- Let $\Gamma_{q}^{1}$ be the graph with: $V\left(\Gamma_{q}^{1}\right):=V\left(\Gamma_{q}-H\right)$ and $E\left(\Gamma_{q}^{1}\right):=E\left(\Gamma_{q}-H\right) \cup \bigcup_{Z \in \mathcal{Z}} M_{Z}$.

- Define $\Gamma_{q}^{2}$ as $V\left(\Gamma_{q}^{2}\right):=V\left(\Gamma_{q}^{1}\right)$ and $E\left(\Gamma_{q}^{2}\right):=\left(E\left(\Gamma_{q}^{1}\right) \backslash\left\{u_{0} v_{0}, u_{1} v_{1}, u_{2} v_{2}\right\}\right) \cup\left\{s_{0} u_{0}, s_{0} v_{0}\right.$, $\left.s_{1} u_{1}, s_{1} v_{1}, s_{2} u_{2}, s_{2} v_{2}\right\}$, the deleted edges $u_{i} v_{i}$ belong to $M_{X_{i}}$ in $\Gamma_{q}^{1}$ and they are replaced by the paths of length two $u_{i} s_{i} v_{i}, i \in\{0,1,2\}$.

Remark 10. Note that $\left|V\left(\Gamma_{q}^{1}\right)\right|=\left|V\left(\Gamma_{q}^{2}\right)\right|=\left|V\left(\Gamma_{q}\right)\right|-3(q+1)+1$. All vertices in $\Gamma_{q}^{1}$ have degree $q+1$ except for $s_{0}, s_{1}, s_{2}$ which remain of degree $q-1$. Hence, by Definition 9 , in $\Gamma_{q}^{2}$ all vertices are left with degree $q+1$. From Equation (1) the girth of both $\Gamma_{q}^{1}$ and $\Gamma_{q}^{2}$ is at most 7 .

Proposition 11. Let $\Gamma_{q}$ be a $(q+1,8)$-cage for odd prime power $q \geqslant 5$ and $\Gamma_{q}^{1}$, $\Gamma_{q}^{2}$ be as in Definition 9.

(a) $\Gamma_{q}^{1}$ has girth 7 if the matchings $M_{S_{i}}, M_{X_{i}}$ and $M_{Y_{i}}$ have the following properties:

(a1) For all $u v \in M_{S_{i}}, E\left(\Gamma_{q}^{1}\left[N_{2}(u v) \cap S_{j}\right]\right) \cap M_{s_{j}}=\emptyset$.

(a2) For all $u v \in M_{X_{i}}, E\left(\Gamma_{q}^{1}\left[N_{2}(u v) \cap Y_{j}\right]\right) \cap M_{Y_{j}}=\emptyset$.

(b) If both conditions (a1) and (a2) hold, the graph $\Gamma_{q}^{2}$ also has girth $\%$.

Proof. By Remark 10 the graphs $\Gamma_{q}^{1}$ and $\Gamma_{q}^{2}$ have girth at most 7. From Remark 4, the distances in $\Gamma_{q}-H$ between the elements in the sets $Z \in \mathcal{Z}$ satisfy the following (see Figure 2):

(i) If $u, v \in Z$, then $d_{\Gamma_{q}-H}(u, v) \geqslant 6$.

(ii) If $u \in X_{i}$ and $v \in X_{j}$, then $d_{\Gamma_{q}-H}(u, v) \geqslant 4$. 
(iii) If $u \in Y_{i}$ and $v \in Y_{j}$, then $d_{\Gamma_{q}-H}(u, v) \geqslant 4$.

(iv) If $u \in S_{i}$ and $v \in S_{j}$, then it may exist $w \in \Gamma_{q}-H$ such that $u, v \in N(w)$, that is, $d_{\Gamma_{q}-H}(u, v) \geqslant 2$.

(v) If $u \in S_{i}$ and $v \in X_{j} \cup Y_{j}$, then $d_{\Gamma_{q}-H}(u, v) \geqslant 3$.

(vi) If $u \in X_{i}$ and $v \in Y_{j}$, then $d_{\Gamma_{q}-H}(u, v) \geqslant 2$.

Let $C$ be a shortest cycle in $\Gamma_{q}^{1}$. If $E(C) \subset E\left(\Gamma_{q}-H\right)$, then $|C| \geqslant 8$. Suppose $C$ contains edges in $M=\bigcup_{Z \in \mathcal{Z}} M_{Z}$. If $C$ contains exactly one such edge, then by (i), $|C| \geqslant 7$. If $C$ contains exactly two edges $e_{1}, e_{2} \in M$, the following cases arise:

- If both $e_{1}, e_{2}$ lie in the same $M_{Z}$, then by (i), $|C| \geqslant 14>7$.

- If $e_{1} \in M_{X_{i}}$ and $e_{2} \in M_{X_{j}}$ for $i \neq j$, by (ii), $|C| \geqslant 10>7$.

- If $e_{1} \in M_{Y_{i}}$ and $e_{2} \in M_{Y_{j}}$ for $i \neq j$, by (iii), $|C| \geqslant 10>7$.

- If $e_{1} \in M_{S_{i}}$ and $e_{2} \in M_{X_{j}} \cup M_{Y_{j}}$, by (v), $|C| \geqslant 8>7$.

- If $e_{1} \in M_{S_{i}}$ and $e_{2} \in M_{S_{j}}$ for $i \neq j$, by item $(a 1),|C| \geqslant 7$.

- If $e_{1} \in M_{X_{i}}$ and $e_{2} \in M_{Y_{j}}$, by item $(a 2),|C| \geqslant 7$.

If $C$ contains at least three edges of $M$, since $d(u, v) \geqslant 2$ for all $u, v \in\left\{X_{i} \cup Y_{i}\right\}_{i=1}^{k} \cup\left\{S_{i}\right\}_{i=4}^{k}$, $|C| \geqslant 9>7$. Hence $\Gamma_{q}^{1}$ has girth 7 , concluding the proof of $(a)$.

To prove (b), let $C$ be a shortest cycle in $\Gamma_{q}^{2}$. If $E(C) \subset E\left(\Gamma_{q}-H\right) \cup M$, then $|C| \geqslant 7$.

- If $C$ contains exactly one edge $s_{i} u_{i}$ or $s_{i} v_{i}$, then $|C| \geqslant 7$ since $d_{\Gamma_{q}}\left(s_{i}, u_{i}\right)=d_{\Gamma_{q}}\left(s_{i}, v_{i}\right)=$ 2 which implies $d_{\Gamma_{q}^{1}}\left(s_{i}, u_{i}\right) \geqslant 6$ and $d_{\Gamma_{q}^{1}}\left(s_{i}, v_{i}\right) \geqslant 6$.

- If $C$ contains a path $u_{i} s_{i} v_{i}$, then $\left(C-\left\{s_{i}\right\}\right)+u_{i} v_{i}$ is a cycle in $\Gamma_{q}^{1}$ with one vertex less than $C$, therefore $|C| \geqslant 8$.

- If $C$ contains two edges $s_{i} u_{i}, s_{j} u_{j}$, for $i \neq j$, their distances $d_{\Gamma_{q}^{1}}\left(s_{i}, u_{j}\right) \geqslant 4$, $d_{\Gamma_{q}^{1}}\left(s_{i}, s_{j}\right) \geqslant 4$, and $d_{\Gamma_{q}^{1}}\left(u_{i}, u_{j}\right) \geqslant 4$.

Since in either case $C$ has length at least 7 and by Remark 10, the result holds.

The following lemma states the existence of the matchings $M_{S_{i}}$ for the sets $S_{i}$, which fulfill condition (a1) from Proposition 11. Notice that in the incidence graph of a generalized quadrangle $\{x, y\}^{\perp \perp}=\bigcap_{s \in N_{2}(x) \cap N_{2}(y)} N_{2}(s)$, thus Remark 1 implies that $\left|\bigcap_{i=0}^{q} N\left(S_{i}\right)\right|=$ $q-1$, recalling that $\left\{s_{i}\right\}_{i=0}^{q}=N_{2}(x) \cap N_{2}(y)$. Since $\left|\bigcap_{i=0}^{q} N\left(S_{i}\right)\right|$ is contained in $\left|\bigcap_{i=3}^{q} N\left(S_{i}\right)\right|$, and $\left|\bigcap_{i=3}^{q} N\left(S_{i}\right)\right|=q-1$, then the condition for the following lemma holds. 
Lemma 12. There exist matchings $M_{S_{i}}$, for $i=3, \ldots, q$, such that condition (a1) in Proposition 11 holds.

Proof. From the regularity of $W(q)$ we know that $\bigcap_{i=0}^{q} N\left(S_{i}\right)=\left\{w_{1}, \ldots, w_{q-1}\right\}$, and since $S_{i}$ has $q-1$ vertices, every vertex $w_{j}$ is adjacent to exactly one vertex in $s_{i j} \in S_{i}$. Moreover, note that $s_{i j}$ is well labeled, because if $s_{i j}$ had two neighbors $w_{j}, w_{j^{\prime}} \in \bigcap_{i=0}^{q} N\left(S_{i}\right), \Gamma_{q}$ would contain the cycle $\left(s_{i j} w_{j} s_{k j} s_{k} s_{k j^{\prime}} w_{j^{\prime}}\right)$ of length 6 .

Therefore, take the complete graph $K_{q-1}$, label its vertices as $j \in\{1, \ldots, q-1\}$. We know that it has a 1 -factorization with $q-2$ factors $F_{1}, \ldots, F_{q-2}$ since $q-1$ is even. For each $i=3, \ldots, q+1$, let $s_{i j} s_{i l} \in M_{S_{i}}$ if and only if $j l \in F_{i-2}$.

To prove that the matchings $M_{S_{i}}$ defined in this way fulfill the desired property suppose that $s_{i j} s_{i l} \in M_{S_{i}}$ and $s_{i^{\prime} j} s_{i^{\prime} l} \in M_{S_{i}^{\prime}}$ for $i^{\prime} \neq i$. Then $F_{i}$ and $F_{i^{\prime}}$ would have the edge $j l$ in common contradicting that they were a factorization.

So far, our construction has been independent from the coordinatization of the chosen $(q+1,8)$-cage, however, in order to define $M_{X_{i}}$ and $M_{Y_{i}}$ satisfying condition $(a 2)$ of Proposition 11, we need to fix all the elements chosen so far. To this purpose we use the following convenient description of a $(q+1,8)$-cage.

Definition 13. $[1,2]$ Let $\mathbb{F}_{q}$ be a finite field with $q \geqslant 2$ a prime power and $\varrho$ a symbol not belonging to $\mathbb{F}_{q}$. Let $\Gamma_{q}=\Gamma_{q}\left[V_{0}, V_{1}\right]$ be a bipartite graph with vertex sets $V_{i}=$ $\mathbb{F}_{q}^{3} \cup\left\{(\varrho, b, c)_{i},(\varrho, \varrho, c)_{i}: b, c \in \mathbb{F}_{q}\right\} \cup\left\{(\varrho, \varrho, \varrho)_{i}\right\}, i=0,1$, and edge set defined as follows:

For all $a \in \mathbb{F}_{q} \cup\{\varrho\}$ and for all $b, c \in \mathbb{F}_{q}$ :

$$
\begin{aligned}
& N_{\Gamma_{q}}\left((a, b, c)_{1}\right)= \begin{cases}\left\{\left(w, a w+b, a^{2} w+2 a b+c\right)_{0}: w \in \mathbb{F}_{q}\right\} \cup\left\{(\varrho, a, c)_{0}\right\} & \text { if } a \in \mathbb{F}_{q} ; \\
\left\{(c, b, w)_{0}: w \in \mathbb{F}_{q}\right\} \cup\left\{(\varrho, \varrho, c)_{0}\right\} & \text { if } a=\varrho .\end{cases} \\
& N_{\Gamma_{q}}\left((\varrho, \varrho, c)_{1}\right)=\left\{(\varrho, c, w)_{0}: w \in \mathbb{F}_{q}\right\} \cup\left\{(\varrho, \varrho, \varrho)_{0}\right\} \\
& N_{\Gamma_{q}}\left((\varrho, \varrho, \varrho)_{1}\right)=\left\{(\varrho, \varrho, w)_{0}: w \in \mathbb{F}_{q}\right\} \cup\left\{(\varrho, \varrho, \varrho)_{0}\right\} .
\end{aligned}
$$

Or equivalently

For all $i \in \mathbb{F}_{q} \cup\{\varrho\}$ and for all $j, k \in \mathbb{F}_{q}$ :

$$
\begin{aligned}
& N_{\Gamma_{q}}\left((i, j, k)_{0}\right)= \begin{cases}\left\{\left(w, j-w i, w^{2} i-2 w j+k\right)_{1}: w \in \mathbb{F}_{q}\right\} \cup\left\{(\varrho, j, i)_{1}\right\} & \text { if } i \in \mathbb{F}_{q} ; \\
\left\{(j, w, k)_{1}: w \in \mathbb{F}_{q}\right\} \cup\left\{(\varrho, \varrho, j)_{1}\right\} & \text { if } i=\varrho .\end{cases} \\
& N_{\Gamma_{q}}\left((\varrho, \varrho, k)_{0}\right)=\left\{(\varrho, w, k)_{1}: w \in \mathbb{F}_{q}\right\} \cup\left\{(\varrho, \varrho, \varrho)_{1}\right\} ; \\
& N_{\Gamma_{q}}\left((\varrho, \varrho, \varrho)_{0}\right)=\left\{(\varrho, \varrho, w)_{1}: w \in \mathbb{F}_{q}\right\} \cup\left\{(\varrho, \varrho, \varrho)_{1}\right\} .
\end{aligned}
$$

Lemma 14. There exist matchings $M_{X_{i}}$ and $M_{Y_{i}}$, for $i=0, \ldots, q$, such that condition (a2) in Proposition 11 holds. 
Proof. Let $x=(\varrho, \varrho, \varrho)_{1}$ and $y=(0,0,0)_{1}$.

We will distinguish two cases, when $q=p$ is a prime or when $q=p^{a}, a>1$ is a prime power.

Case 1: $q=p$ a prime.

Let $x_{i}=(\varrho, \varrho, i)_{0}, y_{i}=(i, 0,0)_{0}$ for $i=0, \ldots, p-1$, and $x_{p}=(\varrho, \varrho, \varrho)_{0}, y_{p}=(\varrho, 0,0)_{0}$.

$N\left(x_{i}\right)=\left\{(\varrho, t, i)_{1}: t=0, \ldots, p-1\right\} \cup\{x\}, i=\{0, \ldots, p-1\}$,

$N\left(x_{p}\right)=\left\{(\varrho, \varrho, t)_{1}: t=0, \ldots, p-1\right\} \cup\{x\}$,

$N\left(y_{i}\right)=\left\{\left(t,-i t, i t^{2}\right)_{1}: t=0, \ldots, p-1\right\} \cup\left\{(\varrho, 0, i)_{1}\right\}, i=\{0, \ldots, p-1\}$,

and $N\left(y_{p}\right)=\left\{(0, t, 0)_{1}: t=0, \ldots, p-1\right\} \cup\left\{(\varrho, \varrho, 0)_{1}\right\}$.

Hence, we have the sets:

$X_{i}=\left\{(\varrho, t, i)_{1}: t=1, \ldots, p-1\right\}$ and $Y_{i}=\left\{\left(t,-i t, i t^{2}\right)_{1}: t=1, \ldots, p-1\right\}$ for $i=0, \ldots, p-1$.

$X_{p}=\left\{(\varrho, \varrho, t)_{1}: t=1, \ldots, p-1\right\}$ and $Y_{p}=\left\{(0, t, 0)_{1}: t=1, \ldots, p-1\right\}$.

Define the following matchings, depicted in Figure 3:

$M_{X_{i}}=\left\{(\varrho, \ell, i)_{1}(\varrho,-(\ell+2), i)_{1}: \ell=1, \ldots,(p-3) / 2\right\} \cup\left\{(\varrho,-2, i)_{1}(\varrho,-1, i)_{1}\right\}$, and

$M_{Y_{i}}=\left\{\left(t,-i t, i t^{2}\right)_{1}\left(-t, i t, i t^{2}\right)_{1}: t=1, \ldots,(p-1) / 2\right\}$, for $i=0, \ldots, p-1$.

$M_{X_{p}}=\left\{(\varrho, \varrho, \ell)_{1}(\varrho, \varrho,-(\ell+2))_{1}: \ell=1, \ldots,(p-3) / 2\right\} \cup\left\{(\varrho, \varrho,-2)_{1}(\varrho, \varrho,-1)_{1}\right\}$, and $M_{Y_{p}}=\left\{(0, t, 0)_{1}(0,-t, 0)_{1}: t=1, \ldots,(p-1) / 2\right\}$.

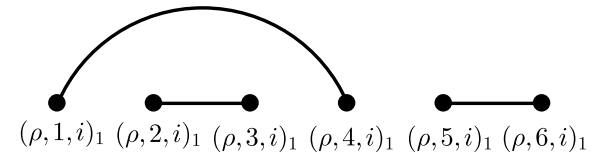

$M_{X_{i}}$

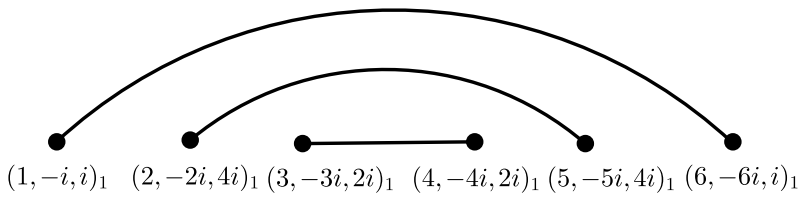

$M_{Y_{i}}$

Figure 3: The matchings $M_{X_{i}}$ and $M_{Y_{i}}$ for $i=0, \ldots, p-1$ and $p=7$, determined by the $2^{\text {nd }}$ and the $1^{\text {rst }}$ coordinates of the vertices in $X_{i}$ and $Y_{i}$ respectively.

Claim: Matchings $M_{X_{j}}$ and $M_{Y_{i}}$ for $i, j \in\{0, \ldots, p\}$, satisfy property (a2). To prove it we must analyze the intersection of the second neighborhood of $X_{j}$ with $Y_{i}$.

Recall that the vertices in $Y_{i}$ are in $N\left((i, 0,0)_{0}\right)=N\left(y_{i}\right)$, the vertices in $X_{j}$ have coordinates $(\varrho, \ell, j)_{1}$ for $\ell=1, \ldots, p-1$. We will show that there exists a unique vertex

$$
w_{i \ell j} \in Y_{i} \cap N_{2}\left(X_{j}\right) \cap N_{2}\left(X_{p}\right)
$$

and to this purpose we describe the coordinates of the vertices in $X_{p}$ in terms of the subscripts of $w_{i \ell j}$ as $\left(\varrho, \varrho, s_{i \ell j}\right)_{1}$ where $s_{i \ell j} \in\{1, \ldots, p-1\}$ and where the relationship between $w_{i \ell j}$ and $s_{i \ell j}$ is highlighted in what follows:

Note that $w_{i \ell j}=(a, b, c)_{1} \in N\left((i, 0,0)_{0}\right) \cap N_{2}\left((\varrho, \ell, j)_{1}\right) \cap N_{2}\left(\left(\varrho, \varrho, s_{i \ell j}\right)_{1}\right)$.

Since $N\left((i, 0,0)_{0}\right)=\left\{\left(t,-i t, i t^{2}\right)_{1}: t=0, \ldots, p-1\right\} \cup(\varrho, 0, i)_{1}$, then $a=t, b=-i t$, and $c=i t^{2}$. Also, $N_{2}\left(\left(\varrho, \varrho, s_{i \ell j}\right)_{1}\right)=N\left(\left\{\left(\varrho, s_{i \ell j}, x\right)_{0}: x \in 0, \ldots, p-1\right\}\right)=\left\{\left(s_{i \ell j}, u, x\right)_{1}\right.$ : $x, u \in 0, \ldots, p-1\}$. Hence, $s_{i \ell j}=a=t$ and $(a, b, c)_{1}=\left(s_{i \ell j},-i s_{i \ell j}, i s_{i \ell j}^{2}\right)_{1}$. Moreover, 
$N\left((\varrho, \ell, j)_{1}\right)=\left\{(j, \ell, s)_{0}: s=0, \ldots, p-1\right\} \cup\left\{(\varrho, \varrho, j)_{0}\right\} ;$ and $N_{2}\left((\varrho, \ell, j)_{1}\right)=N\left(\left\{(j, \ell, s)_{0}:\right.\right.$ $\left.s=0, \ldots, p-1\}) \cup\left\{N(\varrho, \varrho, j)_{0}\right)\right\}=\left\{\left(x, \ell-x j, \ell x^{2}-2 x j+s\right)_{1}\right\} \cup\left\{(\varrho, \ell, j)_{1}\right\}$. By substitution in the first and second coordinates we obtain the following equation:

$$
s_{i \ell j}(j-i)=\ell \text { for } i, \ell, j \in \mathbb{F}_{p}, \ell \neq 0, j-i \neq 0 .
$$

Notice that this equation is undefined for $j=i$, otherwise it would mean that $y_{i}$ has a neighbor at distance 3 from $x_{j}$ and this would imply the existence of a cycle of length 6 in $\Gamma_{q}$.

By equation (5) we have $s_{i(-\ell) j}=-s_{i \ell j}$ implying, for fixed $i$ and $j$, that the vertices $w_{i \ell j}=\left(s_{i \ell j},-i s_{i \ell j}, i s_{i \ell j}^{2}\right)_{1}$ and $w_{i-\ell j}=\left(-s_{i \ell j}, i s_{i \ell j}, i s_{i \ell j}^{2}\right)_{1}$ in $Y_{i}$ are at distance two, respectively, only from the vertices $(\varrho, \ell, j)_{1}$ and $(\varrho,-\ell, j)_{1}$ in $X_{j}$ for $j=\{1, \ldots, p\}$; and to the vertices $(\varrho, \varrho, \ell)$ and $(\varrho, \varrho,-\ell)$ in $X_{p}$. Therefore the matchings $M_{Y_{i}}$ and $M_{X_{j}}$ for $i \in\{1, \ldots, p-1\}$ and $j \in\{1, \ldots, p\}$ satisfy property $(a 2)$.

Finally, also the matchings $M_{X_{j}}$ and $M_{Y_{p}}$ satisfy property $(a 2)$, since for all $(\varrho, \ell, j)_{1} \in$ $X_{j}$ it holds that $Y_{p} \cap N_{2}\left((\varrho, \ell, j)_{1}\right)=(0, \ell, 0)_{1}$; and the edge $(\varrho, \ell, j)_{1}(\varrho,-\ell, j)_{1} \notin M_{X_{j}}$. This ends the proof of the claim.

Case 2: $q$ a prime power.

Let $\alpha$ be a primitive root of unity in $G F(q)=\left\{\alpha^{i}: i=0, \ldots, q-2\right\} \cup\{0\}$.

In this case, $x_{i}=\left(\varrho, \varrho, \alpha^{i-1}\right)_{0}, y_{i}=\left(\alpha^{i-1}, 0,0\right)_{0}$, for $i=1, \ldots, q-1, x_{0}=(\varrho, \varrho, 0)_{0}$, $y_{0}=(0,0,0)_{0}, x_{q}=(\varrho, \varrho, \varrho)_{0}$ and $y_{q}=(\varrho, 0,0)_{0}$.

$N\left(x_{i}\right)=\left\{\left(\varrho, \alpha^{t}, \alpha^{i-t}\right)_{1}: t=0, \ldots, q-2\right\} \cup\left(\varrho, 0, \alpha^{i-1}\right)_{1} \cup x$

$N\left(x_{0}\right)=\left\{\left(\varrho, \alpha^{t}, 0\right)_{1}: t=0, \ldots, q-2\right\} \cup(\varrho, 0,0)_{1} \cup x$,

$N\left(x_{q}\right)=\left\{\left(\varrho, \varrho, \alpha^{t}\right)_{1}: t=0, \ldots, q-2\right\} \cup(\varrho, \varrho, 0)_{1} \cup x$.

$N\left(y_{i}\right)=\left\{\left(\alpha^{t},-\alpha^{i-1+t}, \alpha^{i-1+2 t}\right)_{1}: t=0, \ldots, q-2\right\} \cup\left(\varrho, 0, \alpha^{i-1}\right)_{1}$,

$N\left(y_{0}\right)=\left\{\left(\alpha^{t}, 0,0\right)_{1}: t=0, \ldots, q-2\right\} \cup(\varrho, 0,0)_{1}$,

$N\left(y_{q}\right)=\left\{\left(0, \alpha^{t}, 0\right)_{1}: t=0, \ldots, q-2\right\} \cup(\varrho, \varrho, 0)_{1} \cup y$.

Hence, we have the sets:

$X_{i}=\left\{\left(\varrho, \alpha^{t}, \alpha^{i-1}\right)_{1}: t=0, \ldots, q-2\right\}$ and $Y_{i}=\left\{\left(\alpha^{t},-\alpha^{i-1+t}, \alpha^{i-1+2 t}\right)_{1}: t=0, \ldots, q-\right.$ $2\}$ for $i=1, \ldots, q-1$.

$X_{0}=\left\{\left(\varrho, \alpha^{t}, 0\right)_{1}: t=0, \ldots, q-2\right\}$ and $Y_{0}=\left\{\left(\alpha^{t}, 0,0\right)_{1}: t=0, \ldots, q-2\right\}$.

$X_{q}=\left\{\left(\varrho, \varrho, \alpha^{t}\right)_{1}: t=0, \ldots, q-2\right\}$ and $Y_{q}=\left\{\left(0, \alpha^{t}, 0\right)_{1}: t=0, \ldots, q-2\right\}$.

In order to define the matchings $M_{X_{i}}$ and $M_{Y_{i}}$ we proceed as above, but in this case we obtain the following equations:

$$
\begin{array}{cc}
\alpha^{s_{i \ell j}}\left(\alpha^{j-1}-\alpha^{i-1}\right)=\alpha^{\ell} & \text { for } i, j \geqslant 1 ; \\
\alpha^{s_{0 \ell j}}\left(\alpha^{j-1}\right)=\alpha^{\ell} & \text { for } i=0 ; \\
\alpha^{s_{i \ell 0}}\left(-\alpha^{i-1}\right)=\alpha^{\ell} & \text { for } j=0 .
\end{array}
$$

Notice that equation (6) is undefined for $j=i$, otherwise it would mean that $y_{i}$ has a neighbor at distance 3 from $x_{j}$ and this would imply the existence of a cycle of length 6 in $\Gamma_{q}$.

Define the following matchings, depicted in Figure 4:

$$
M_{X_{i}}=\left\{\left(\varrho, \alpha^{2 \ell}, i\right)_{1}\left(\varrho, \alpha^{2 \ell+1}, i\right)_{1}: \ell=0, \ldots,(q+1) / 2\right\} ; \text { and }
$$




$$
M_{Y_{i}}=\left\{\left(\alpha^{2 t},-\alpha^{i-1+2 t}, \alpha^{i-1+4 t}\right)_{1}\left(\alpha^{2 t+3},-\alpha^{i-1+(2 t+3)}, \alpha^{i-1+2(2 t+3)}\right)_{1}: t=0, \ldots,(q-\right.
$$
1) $/ 2\} \cup\left\{\left(\alpha,-\alpha^{i}, \alpha^{i+1}\right)_{1}\left(\alpha^{q-3},-\alpha^{i+q-4}, \alpha^{i+2 q-7}\right)\right\}$, for $i=0, \ldots, q-1$.

$$
\begin{aligned}
& M_{X_{q}}=\left\{\left(\varrho, \varrho, \alpha^{2 \ell}\right)_{1}\left(\varrho, \varrho, \alpha^{2 \ell+1}\right)_{1}: \ell=0, \ldots,(q+1) / 2\right\} ; \text { and } \\
& M_{Y_{q}}=\left\{\left(0, \alpha^{2 t}, 0\right)_{1}\left(0, \alpha^{2 t+3}, 0\right)_{1}: t=0, \ldots,(q-1) / 2\right\} \cup\left\{(0, \alpha, 0)_{1},\left(0, \alpha^{q-3}, 0\right)_{1}\right\} .
\end{aligned}
$$
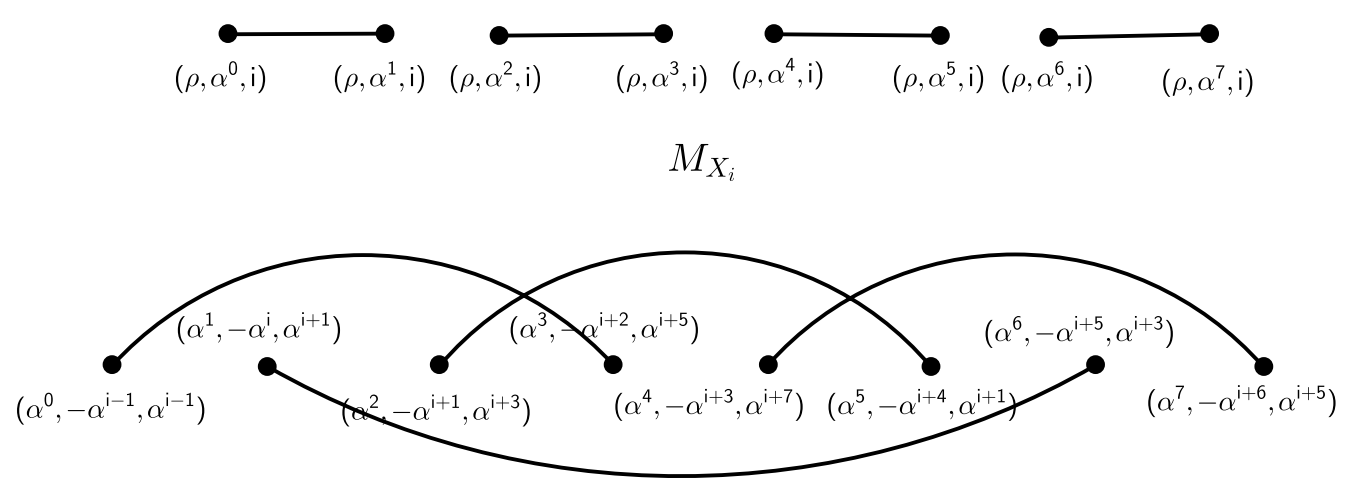

$M_{Y_{i}}$

Figure 4: The matchings $M_{X_{i}}$ and $M_{Y_{i}}$ for $i=0, \ldots, q-1$ and $q=9$, determined by the $2^{\text {nd }}$ and the $1^{r s t}$ coordinates of the vertices in $X_{i}$ and $Y_{i}$ respectively.

On the other hand, we obtain that $s_{i \ell+1 j}=s_{i \ell j}+1$, multiplying the equation (6) by $\alpha$, which implies that the matchings $M_{X_{i}}$ and $M_{Y_{i}}$ satisfy property (a2), because the two vertices $\left(\alpha^{s_{i \ell j}},-\alpha^{i-1+s_{i \ell j}}, \alpha^{i-1+2 s_{i \ell j}}\right)_{1}$ and $\left(\alpha^{s_{i \ell j}+1},-\alpha^{i-1+\left(s_{i \ell j}+1\right)}, \alpha^{i-1+2\left(s_{i \ell j}+1\right)}\right)_{1}$ in $Y_{i}$, are at distance two in $X_{j}$ only from the vertices $\left(\varrho, \alpha^{\ell}, j\right)_{1}$ and $\left(\varrho, \alpha^{\ell+1}, j\right)_{1}$, concluding the proof.

Construction 2: For $q \geqslant 5$ an odd prime power let $\Gamma_{q}^{2}$ be the graph of order $2 q^{3}+$ $2 q^{2}-q+1$ given in Definition 9, with the choice of matchings as in Lemmas 12 and 14 . Then, the graph $\Gamma_{q}^{2}$ is a $(q+1)$-regular graph of girth 7 with $2 q^{3}+2 q^{2}-q+1$ vertices as we prove in the following theorem.

Theorem 15. Let $q \geqslant 5$ be an odd prime power. Then, there is a $(q+1)$-regular graph of girth 7 and order $2 q^{3}+2 q^{2}-q+1$.

Proof. Consider the graph in Construction 2, obtained with the choice of matchings from Lemmas 12 and 14. Then, condition (a) in Proposition 11 holds, and by Proposition 11(b) the graph $\Gamma_{q}^{2}$ has girth 7 . By Remark 10 , the order of $\Gamma_{q}^{2}$ is $2\left(q^{3}+q^{2}+q+1\right)-(q-3+$ $2(q+2))=2 q^{3}+2 q^{2}-q+1$, as required.

Note that for $q=5$, McKay and Yang found eighty-seven $(6,7)$-graph has 294 vertices, in 2001 [26, 32]. Our $(6,7)$-graph has 296 vertices, but for $q>5$, Theorem 15 improves the previously known upper bounds from Sauer (cf. [33]). 


\section{Acknowledgements}

We would like to thank the Editor and the anonymous reviewers for their insightful comments and suggestions to this paper.

\section{References}

[1] M. Abreu, G. Araujo-Pardo, C. Balbuena, D. Labbate. A Construction of Small $(q-1)$-Regular Graphs of Girth 8, Electron. J. Combin. 22(2) (2015) \#P2.10.

[2] M. Abreu, G. Araujo-Pardo, C. Balbuena, D. Labbate. A formulation of a $(q+1,8)$ cage, (submitted).

[3] M. Abreu, G. Araujo-Pardo, C. Balbuena, D. Labbate. Families of Small Regular Graphs of Girth 5. Discrete Math. 312(18):2832-2842, 2012.

[4] M. Abreu, M. Funk, D. Labbate, V. Napolitano. A family of regular graphs of girth 5. Discrete Math. 308(10):1810-1815, 2008.

[5] M. Abreu, M. Funk, D. Labbate, V. Napolitano. On (minimal) regular graphs of girth 6. Australas. J. Combin. 35:119-132, 2006.

[6] G. Araujo, C. Balbuena, and T. Héger. Finding small regular graphs of girths 6, 8 and 12 as subgraphs of cages. Discrete Math. 310(8):1301-1306, 2010.

[7] G. Araujo-Pardo, C. Balbuena. Constructions of small regular bipartite graphs of girth 6. Networks 57(2):121-127, 2011.

[8] E. Bannai and T. Ito. On finite Moore graphs. J. Fac. Sci. Univ. Tokio, Sect. I A Math 20:191-208, 1973.

[9] C. Balbuena. Incidence matrices of projective planes and other bipartite graphs of few vertices. Siam J. Discrete Math. 22(4):1351-1363, 2008.

[10] C. Balbuena. A construction of small regular bipartite graphs of girth 8. Discrete Math. Theor. Comput. Sci. 11(2): 33-46, 2009.

[11] C. T. Benson. Minimal regular graphs of girth eight and twelve. Canad. J. Math. 18:1091-1094, 1966.

[12] N. Biggs. Algebraic Graph Theory. Cambridge University Press, New York, 1996.

[13] N. Biggs. Construction for cubic graphs with large girth. Electron. J. Combin. 5(1) (1998) \#A1.

[14] J. A. Bondy and U. S. R. Murty. Graph Theory. Springer Series: Graduate Texts in Mathematics, Vol. 244, 2008.

[15] G. Brinkmann, B. D. McKay and C. Saager. The smallest cubic graphs of girth nine. Combin. Prob. and Computing 5:1-13, 1995.

[16] P. Erdős and H. Sachs. Reguläre Graphen gegebener Taillenweite mit minimaler Knotenzahl. Wiss. Z. Uni. Halle (Math. Nat.), 12:251-257, 1963.

[17] G. Exoo. A Simple Method for Constructing Small Cubic Graphs of Girths 14, 15 and 16. Electron. J. Combin., 3(1) \#R30, 1996. 
[18] G. Exoo and R. Jajcay Dynamic cage survey. Electron. J. Combin. (2013) \#DS16.

[19] W. Feit and G. Higman. The non-existence of certain generalized polygons. J. Algebra 1:114-131, 1964.

[20] A. Gács and T. Héger. On geometric constructions of $(k, g)$-graphs Contrib. to Discrete Math. 3(1): 63-80, 2008.

[21] D. A. Holton and J. Sheehan. The Petersen Graph, Chapter 6: Cages. Cambridge University, 1993.

[22] F. Lazebnik and V. A. Ustimenko. Explicit construction of graphs with an arbitrary large girth and of large size. Discrete Appl. Math. 60:275-284, 1995.

[23] F. Lazebnik, V. A. Ustimenko, and A. J. Woldar. New upper bounds on the order of cages. Electron. J. Combin. 4(2) (1997) \#R13.

[24] H. van Maldeghem, Generalized polygons. Monographs in Mathematics, 93, Birkhauser Verlag, Basel, 1998.

[25] X. Marcote, C. Balbuena, I. Pelayo. On the connectivity of cages of girth five, six and eight. Discrete Math. 307(11-12):1441-1446, 2007.

[26] B. D. McKay, pers. comm. April 9, 2015.

[27] M. Meringer. Fast generation of regular graphs and construction of cages. J. Graph Theory 30:137-146, 1999.

[28] M. O'Keefe and P. K. Wong. The smallest graph of girth 6 and valency 7. J. Graph Theory 5(1):79-85, 1981.

[29] S.E. Payne. Affine representation of generalized quadrangles. J. Algebra 51:473-485, 1970.

[30] S. E. Payne and J. A. Thas. Finite generalized quadrangles. Research Notes in Mathematics, 110. Pitman (Advanced Publishing Program), Boston, MA, 1984.

[31] T. Pisanski, M. Boben, D. Marusic, A. Orbanic, A. Graovac. The 10-cages and derived configurations. Discrete Math. 275:265-276, 2004.

[32] G. Royle, "Cages of Higher Valency." http://school.maths. uwa.edu.au/ gordon/remote/cages/allcages.html

[33] N. Sauer. Extremaleigenschaften regulärer Graphen gegebener Taillenweite, I and II, Sitzungsberichte - Österreich. Akad. Wiss. Math. Natur. Kl., S-B II 176:9-25, 1967; 176:27-43, 1967.

[34] J. Tits. Sur la trialité et certains groupes qui s'en déduisent. Publ. Math. I.H.E.S. Paris, 2:14-60, 1959.

[35] W. T. Tutte. A family of cubical graphs. Math. Proc. Cambridge Philos. Soc., 43(04):459-474, 1947.

[36] V. A. Ustimenko. A linear interpretation of the flag geometries of Chevalley groups. Ukr. Mat. Zh., Kiev University, 42(3):383-387, 1990. 
[37] V. A. Ustimenko. On the embeddings of some geometries and flag systems in Lie algebras and superalgebras, in: Root Systems, Representation and Geometries. Akad. Nauk Ukrain. SSR Inst. Mat., Preprint 8:3-17, 1990.

[38] W. D. Wallis. One-Factorizations. Kluwer, Dordrecht, 1997.

[39] P. K. Wong. Cages-a survey. J. Graph Theory 6:1-22, 1982. 


\section{Corrigendum - submitted 21 June 2016}

A small arithmetic mistake in quoting Remark 10 in Theorem 15, led to the wrong computation of the number of vertices in our graphs for odd prime powers $q \geqslant 5$, which turn out to be one vertex better than originally stated. The corrections are the following:

Page 13 line -5 : replace " $(q-3+2(q+2))$ " in the formula, by " $(3(q+1)-1)$ " as in Remark 10, which is being quoted at that point.

Replace " $2 q^{3}+2 q^{2}-q+1$ " by " $2 q^{3}+2 q^{2}-q$ " in the following places:

- Abstract page 1 line -3

- page 3 line 12

- page 7 line 7

- page 13 line $-11,-8,-4$. 